\title{
Erratum to: Autophagy is a major regulator of beta cell insulin homeostasis
}

\author{
Yael Riahi $^{1}$ - Jakob D. Wikstrom ${ }^{1,2}$ - Etty Bachar-Wikstrom ${ }^{1} \cdot$ Nava Polin $^{1}$. \\ Hava Zucker ${ }^{1}$ • Myung-Shik Lee ${ }^{3}$ - Wenying Quan ${ }^{4}$ Leena Haataja ${ }^{5}$. \\ Ming Liu ${ }^{5} \cdot$ Peter Arvan $^{5} \cdot$ Erol Cerasi $^{1} \cdot$ Gil Leibowitz $^{1}$
}

Published online: 17 May 2016

(C) Springer-Verlag Berlin Heidelberg 2016

\section{Erratum to: Diabetologia DOI 10.1007/s00125-016-3868-9}

Problems regarding Fig. 2 have come to light after publication of this paper. Specifically:

\section{Fig. 2a}

For cells expressing PI-GFP and p62-mCherry at time point 0 (top row on the right-hand panel) the PI-GFP (green) and p62mCherry (red) channels were mistakenly reversed in the merged image. This mistake is corrected in the figure shown overleaf and does not affect the colocalisation analysis (Fig. 2b), which was performed on correctly merged images.

In the second row of Fig. 2a the green intensity in the rightmost ('Merge') panel is higher than that in the PI-GFP single channel image to the left. The authors confirmed that they

The online version of the original article can be found at http://dx.doi.org/ 10.1007/s00125-016-3868-9.

Gil Leibowitz

gleib@hadassah.org.il

1 Endocrinology and Metabolism Service, Department of Medicine, Hadassah-Hebrew University Medical Center, PO Box 12000, Jerusalem 91120, Israel

2 Molecular Dermatology Research Group, Unit of Dermatology and Venereology, Department of Medicine, Karolinska Institutet, Stockholm, Sweden

3 Severance Biomedical Research Institute and Department of Internal Medicine, Yonsei University College of Medicine, Seoul, South Korea

4 Department of Medicine, Samsung Medical Center, Sungkyunkwan University School of Medicine, Seoul, South Korea

5 Division of Metabolism, Endocrinology and Diabetes, University of Michigan, Ann Arbor, MI, USA adjusted the brightness and contrast in the merged image for cosmetic reasons after quantifications were performed. The exposure in the merged image is corrected in the amended figure shown overleaf. Colocalisation analyses were performed using identical imaging conditions for the control and different treatments so these changes do not affect the interpretation of the findings. The figure legend has been amended to reflect this.

Modest changes in brightness and contrast were uniformly applied to the images in the amended figure to reduce nonspecific background fluorescence, which was not organellespecific. Unmodified images were used for quantifications. The legend has been amended in line with this.

Fig. 2c

In the bottom row of Fig. 2c, a technical problem meant that the images were cropped differently and were not properly aligned in size, shape or with respect to one another. This is corrected overleaf. To avoid any misinterpretation the images are shown using identical brightness and contrast conditions for all single-channel and merged images.

The p62-mCherry (red) signal in the bottom middle panel of Fig. 2c was selectively enhanced from the original image, contravening the journal's image manipulation policy (http:// www.diabetologia-journal.org/instructionstoauthors. html\#manipulation). The authors have recreated Fig. 2c from scratch. Slight adjustments to intensity and contrast were applied uniformly to the single and merged images; the colour balance remained untouched. All quantifications were performed prior to representative image modifications.

Modest changes in brightness and contrast were uniformly applied to the images in the amended figure to reduce nonspecific background fluorescence, which was not organellespecific, and unmodified images were used for quantifications. The legend has been amended in line with this. 

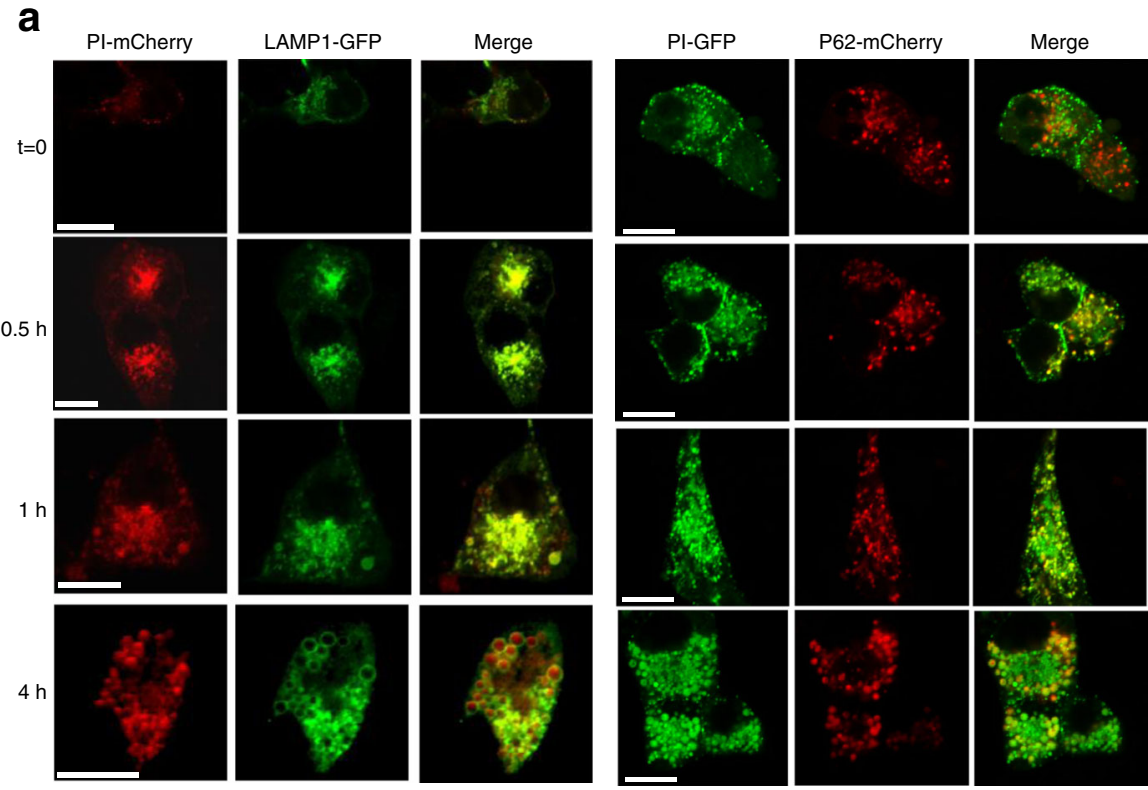

b
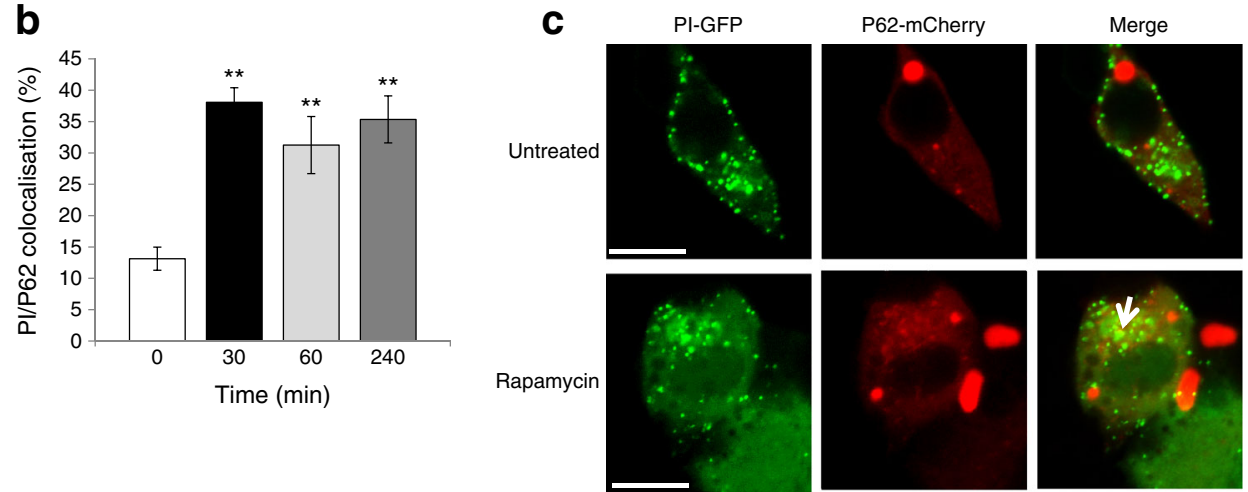

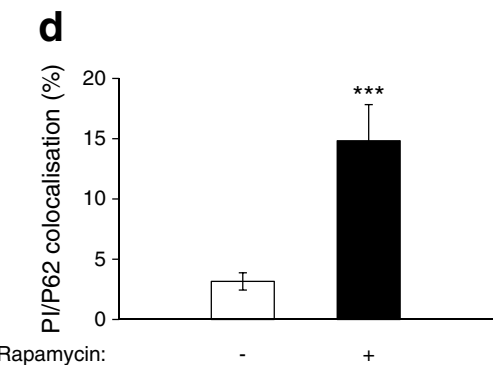

Fig. 2 (a, b) INS-1E beta cells were transfected with WT proinsulin labelled with mCherry or eGFP and with the autolysosome markers LAMP-1-eGFP or with P62-mCherry. After $24 \mathrm{~h}$, cells were treated with bafilomycin-A1 for different times, followed by confocal microscope imaging. (b) Quantification of proinsulin/P62 colocalisation $(n=3)$. (c, d) INS-1E cells were transfected with proinsulin-egfp or proinsulin$m$ Cherry and P62-mCherry and then treated with $50 \mathrm{nmol} / 1$ rapamycin for $16 \mathrm{~h}$. The arrowhead indicates colocalisation in autophagic puncta. The very large mCherry-positive blobs, some of which appear to be extracellular, probably represent artefactual p62-mCherry aggregates. (d) Quantification of proinsulin-P62/SQSTM1 colocalisation $(n=3)$. (e) Cells were incubated with cycloheximide for $2 \mathrm{~h}$ with or without $50 \mathrm{nmol} / 1$ rapamycin and proinsulin content was analysed by ELISA $(n=3$ in triplicate); $p=0.059$. (f, g) INS-1E cells were treated overnight with $100 \mathrm{mmol} / \mathrm{l}$ trehalose or with Tat-Beclin1 $(10 \mu \mathrm{mol} / \mathrm{l})$ for $3.5 \mathrm{~h}$. (f)

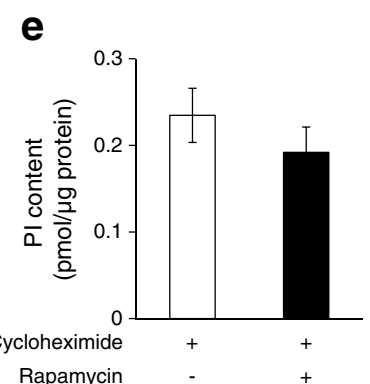

Autophagy was assessed by western blotting for LC3-II in cells treated with or without bafilomycin-A1 $(n=3)$. Stimulation of autophagy is evident by increased accumulation of LC3-II in the presence of bafilomycin-A1. (g) Proinsulin content was analysed by ELISA $(n=3$ in triplicate). (h) INS-1E cells were transfected with proinsulin-egfp, Lc3-mCherry and B4galt1-mTurquoise2 (mTurquoise-Golgi) constructs, then treated with and without bafilomycin-A1. Proinsulin and B4GALT1 localisation in autophagosomes (LC3-eGFP+ punctae) analysed by confocal microscope. Modest changes in brightness and contrast were uniformly applied to all the images to reduce non-specific background fluorescence, which was not organelle-specific. Unmodified images were used for quantifications. Scale bars, $10 \mu \mathrm{m} .{ }^{*} p<0.05,{ }^{*} p<0.01$, $* * * p<0.001$. Baf A1, bafilomycin-A1; C-pep, C-peptide; Ctrl, control; GAPDH, glyceraldehyde 3-phosphate dehydrogenase; PI, proinsulin 